\title{
Results of a phase I pilot clinical trial examining the effect of plant-derived resveratrol and grape powder on Wnt pathway target gene expression in colonic mucosa and colon cancer
}

\author{
Anthony $\vee$ Nguyen' \\ Micaela Martinez' \\ Michael J Stamos ${ }^{2}$ \\ Mary P Moyer ${ }^{3}$ \\ Kestutis Planutis' \\ Christopher Hope' \\ Randall F Holcombe' \\ 'Division of Hematology/Oncology \\ and Chao Family Comprehensive \\ Cancer Center, ${ }^{2}$ Department \\ of Surgery, University of California, \\ Irvine CA, USA; ${ }^{3}$ Incell Corporation, \\ San Antonio, TX USA
}

\begin{abstract}
Context: Resveratrol exhibits colon cancer prevention activity in animal models; it is purported to have this activity in humans and inhibit a key signaling pathway involved in colon cancer initiation, the Wnt pathway, in vitro.

Design: A phase I pilot study in patients with colon cancer was performed to evaluate the effects of a low dose of plant-derived resveratrol formulation and resveratrol-containing freeze-dried grape powder (GP) on Wnt signaling in the colon. Eight patients were enrolled and normal colonic mucosa and colon cancer tissue were evaluated by Wnt pathway-specific microarray and quantitative real-time polymerase chain reaction (qRT-PCR) pre- and post-exposure to resveratrol/GP. Results: Based on the expression of a panel of Wnt target genes, resveratrol/GP did not inhibit the Wnt pathway in colon cancer but had significant $(\mathrm{p}<0.03)$ activity in inhibiting Wnt target gene expression in normal colonic mucosa. The greatest effect on Wnt target gene expression was seen following ingestion of $80 \mathrm{~g}$ of GP per day $(\mathrm{p}<0.001)$. These results were confirmed with qRT-PCR of cyclinD1 and axinII. The inhibitory effect of GP on Wnt signal throughput was confirmed in vitro with a normal colonic mucosa-derived cell line.

Conclusions: These data suggest that GP, which contains low dosages of resveratrol in combination with other bioactive components, can inhibit the Wnt pathway in vivo and that this effect is confined to the normal colonic mucosa. Further study of dietary supplementation with resveratrol-containing foods such as whole grapes or GP as a potential colon cancer preventive strategy is warranted.
\end{abstract}

Trial registration: NCT00256334.

Keywords: resveratrol, clinical trial, colon cancer, Wnt signaling, grapes, cancer prevention

\section{Introduction}

Colon cancer is the third leading cause of cancer deaths among men and women in the United States. ${ }^{1}$ The epidemiology of colon cancer suggests that dietary factors play a critical role in tumor initiation and promotion, ${ }^{2}$ with populations consuming a diet high in fiber, vegetables, and fruits exhibiting a lower cancer incidence. Recent dietary recommendations for cancer prevention highlight the importance of a plant-based diet in the prevention of several types of cancer.,

Resveratrol is a stilbene found in the skin of grapes, red wine, peanuts, and some other plant species. In vitro, this agent has been shown to have antioxidant activity and proapoptotic activity, and to inhibit proliferation of colon cancer cell lines. ${ }^{5}$ The data for prevention of colon cancer by resveratrol in animal models are somewhat contradictory, both in its efficacy as well as information regarding the effective dose range. Tessitore ${ }^{6}$ demonstrated activity of a very low dose resveratrol of $0.2 \mathrm{mg} / \mathrm{kg} /$ day in reducing aberrant crypt foci (ACF) in the colon
Correspondence: Randall F Holcombe Chao Family Comprehensive Cancer Center, University of California, Irvine Medical Center, I0I The City Drive Building 56, Room 247 Orange,

CA 92868, USA

$\mathrm{Tel}+\mathrm{I} 7 \mathrm{I} 44565153$

$\mathrm{Fax}+\mathrm{I} 7144562242$

Email rholcomb@uci.edu 
in an azoxymethane-induced tumor model. In another carcinogen-based model, utilizing 1,2-dimethylhydrazine, resveratrol in a dose of $8 \mathrm{mg} / \mathrm{kg} /$ day reduced both ACF and colonic tumors. ${ }^{7,8}$ In genetic models utilizing the $\mathrm{APC}^{\mathrm{min} /+}$ mouse, which harbors a single allele mutation in $a p c$, and therefore has intrinsically activated Wnt signaling, Schneider ${ }^{9}$ demonstrated profound activity at dosages as low as $0.3 \mathrm{mg} / \mathrm{mouse} /$ day in reducing intestinal tumors. In this study, expression of cyclinD1, a Wnt target gene, as well as other markers of cell cycling, was reduced. Other studies $^{10-12}$ have found resveratrol to be ineffective or effective only at high dosages in inducing a more modest reduction in intestinal tumorigenesis. Overall, these studies indicate that resveratrol may have activity for colon cancer prevention in both carcinogen-induced tumor models as well as in the Wnt-activated $\mathrm{APC}^{\mathrm{min} /+}$ mouse, but that the effective dose is unclear.

Resveratrol is also reported to inhibit ribonucleotide reductase in leukemia cell lines, ${ }^{13}$ DNA polymerase in fibroblasts, ${ }^{14}$ ornithine decarboxylase in mouse hepatic tissue, ${ }^{15}$ and IkappaB kinase in monocyte cell lines and mouse skin. ${ }^{16,17}$ Activation of SIRT1 (sirtuin-1) is thought to be an important mechanism through which resveratrol exerts antiaging activity. ${ }^{18}$ While inhibition of IkappaB kinase and DNA polymerase can be seen at concentrations as low as $1 \mu \mathrm{M}$, SIRT1 activation and inhibition of other target enzymes generally requires concentrations between $50 \mu \mathrm{M}$ and $100 \mu \mathrm{M}$, demonstrating that this compound has differential effects over at least a $100 \times$ concentration range.

In relation to the cancer chemopreventive effects, the concentrations attained in vivo are significantly lower than the concentrations required for inhibition of cell growth in vitro, raising the question as to how resveratrol exhibits this activity in animal models and perhaps in humans. ${ }^{19}$ A recent pharmacokinetic study of single dose resveratrol confirmed that peak plasma concentrations of the parent compound following a single large $5 \mathrm{~g}$ ingestion reached only $539 \mathrm{ng} / \mathrm{ml}$ $(2.4 \mu \mathrm{M}) .{ }^{20}$ However, the peak levels of resveratrol-3-sulfate and two monoglucuronides were three to eight times higher, raising the possibility that cancer prevention activity may be, at least in part, attributable to resveratrol's principal metabolites. Other explanations of resveratrol's in vivo activity may be that, even though serum concentrations are low, local concentrations in the gut are sufficient to provide activity. Alternatively, resveratrol's activity may be augmented by, or at low concentration be dependent upon cooperativity with other bioactive compounds present in dietary sources such as grapes.
The Wnt signaling pathway is critically important in the development of colon cancer, with activating mutations seen in over $85 \%$ of sporadic cases. ${ }^{21}$ Mutations in either apc or $\beta$-catenin are early events which constitutively activate the pathway ${ }^{22}$ promoting cell growth and proliferation and inhibiting normal patterns of colonic stem cell differentiation. ${ }^{23}$ Prior studies have demonstrated that relatively low concentrations $(10 \mu \mathrm{M}-20 \mu \mathrm{M})$ of resveratrol can inhibit Wnt signal throughput in colon-derived cells in vitro. ${ }^{24,25}$ This may be one of the potential mechanisms by which resveratrol provides colon cancer prevention activity in vivo. To evaluate this directly, a phase I pilot clinical trial of low dose, plantderived resveratrol and resveratrol-containing freeze-dried grape powder (GP) was initiated in patients with colon cancer. Our hypothesis was that resveratrol and resveratrol-containing GP would inhibit Wnt signaling in vivo in both the normal colonic mucosa and cancer tissue. This trial, with biomarker endpoints evaluating the expression of multiple components and target genes of the Wnt pathway, represents the first reported clinical trial of resveratrol in patients with cancer. We demonstrate here that both plant-derived resveratrol and GP have effects in vivo on various measures of the Wnt pathway in colon cancer and normal colonic mucosa. Resveratrol in combination with other bioactive components present in GP had a significant effect on suppressing Wnt pathway target gene expression, a measure of Wnt signal throughput, in normal colonic mucosa suggesting their potential incorporation into colon cancer prevention strategies.

\section{Experimental methodology Clinical protocol}

Patients with suspected or documented colorectal cancer were identified through clinics at the H.H. Chao Digestive Diseases Center and the Chao Family Comprehensive Cancer Center at the University of California, Irvine. All patients completed written informed consent and enrolled onto an Institution Review Board-approved clinical trial. The clinical trial was registered with clinicaltrials.gov prior to study initiation (NCT00256334). Between September 22, 2005 and July 5, 2008 , eight patients were enrolled onto the study. Since most patients diagnosed with colon cancer by sigmoidoscopy or colonoscopy do not undergo additional endoscopic examination prior to surgical resection, accrual was hampered by the necessity for obtaining fresh normal and tumor tissue prior to biopsy confirmation of cancer. Charts for over 250 patients scheduled for colonoscopic examination were screened. More than 50 individuals were identified as potential colon cancer patients and were enrolled onto the tissue acquisition 
component of this study. Of these, 11 patients were diagnosed with cancer and eight agreed to participation in the resveratrol/ GP intervention prior to surgery. The initial target enrollment was 12 patients; three patients were to be randomized to four dose cohorts. Because of slow enrollment related to the study design, this study was closed after eight patients completed the intervention. The study was an open label phase I pilot study with biomarker endpoints.

At the time of diagnostic colonoscopy for suspected colon cancer, tissue biopsies were obtained from cancer tissue and normal colonic mucosa. Matched specimens for each were reviewed by pathology to confirm the histologic diagnosis. Tissue was placed into RNAlater ${ }^{\circledR}$ (Applied Biosystems, Foster City, CA) and total RNA was later isolated with a Trizol reagent. Patients then received 14 days of resveratrol or freeze-dried GP until the day prior to surgery for colon cancer resection. The 14-day treatment period was selected because the typical delay from diagnosis to elective surgical resection was 14 to 16 days at our institution. If participation in the study would have necessitated a delay in surgery, then enrollment was not permitted. Patients were randomly assigned to one of four dose cohorts: plant-derived resveratrol tablets (purchased through the Life Extension Foundation, Scottsdale, AZ) at a dose of $80 \mathrm{mg} /$ day, plant-derived resveratrol tablets at a dose of $20 \mathrm{mg}$ /day, GP dissolved in water and taken orally (supplied by the California Table Grape Commission) at a dose of $120 \mathrm{~g} /$ day, and GP at a dose of $80 \mathrm{~g} /$ day (Table 1). This latter dose is equivalent to approximately $0.45 \mathrm{~kg}$ of fresh grapes/day (three United States Department of Agriculture (USDA)- recommended servings/day). At the time of surgical resection, biopsies from the cancer and normal colonic mucosa were obtained and processed identically to the pretreatment specimens.

Resveratrol capsules were obtained from Life Extension Corporation as a plant-derived nutritional supplement containing $20 \mathrm{mg}$ trans-resveratrol per capsule derived from whole red grapes and Polygonum cuspidatum root extracts. Each capsule also had $120 \mathrm{mg}$ of quercetin as quercetin dehydrate. High-pressure liquid chromatography (HPLC) analysis of the $20 \mathrm{mg}$ capsules revealed only $3.886 \mathrm{mg}$ of trans-resveratrol (personal communication, Dr Arthur S Polans, University of Wisconsin). Freeze-dried GP was obtained from the California Table Grape Commission. It is a composite of fresh red, green, and blue-black California grapes (seeded and seedless varieties), that were frozen, ground with food-quality dry ice, freeze-dried, and reground using good manufacturing practices for food products throughout. The powder was processed and

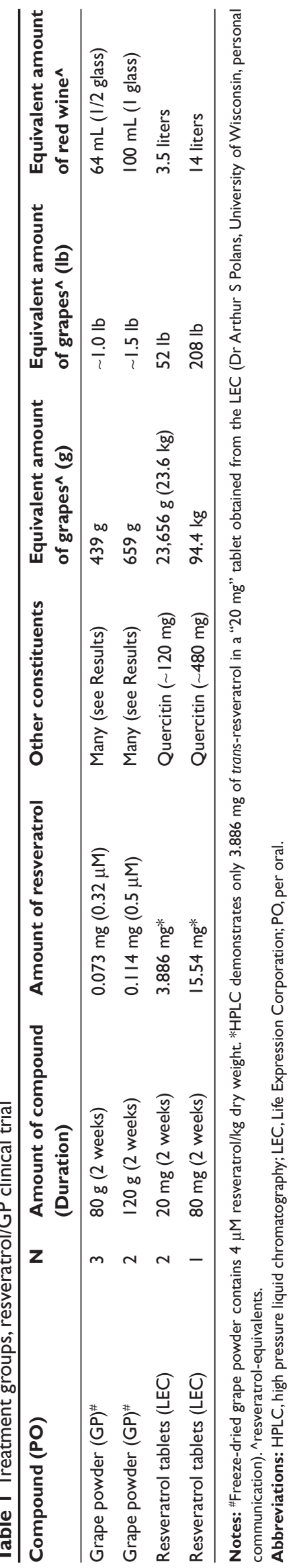


stored to preserve the integrity of the biologically-active compounds found in fresh grapes. As with fresh grapes, the powder is known to contain resveratrol, flavans (including catechin), flavonols (including quercetin), anthocyanins and simple phenolics. Detailed analysis of the GP revealed resveratrol, $4 \mu \mathrm{M} / \mathrm{kg}$ of dry powder; flavonols, $118 \mu \mathrm{M} / \mathrm{kg}$; anthocyanins, $700 \mathrm{mg} / \mathrm{kg}$; flavans, $3.9 \mathrm{mg} / \mathrm{g}$ (as catechin); and total phenols, $6.2 \mathrm{mg} / \mathrm{g}$. Total phenols were analyzed by reaction with Folin and Ciocalteu's phenol reagent; flavans were analyzed by reaction with vanillin; anthocyanins were analyzed spectrophotometrically; flavonols and resveratrol were analyzed by HPLC after acid hydrolysis.

Microarray pre- and post-specimen cDNA was analyzed with a GEArray Q Series Human Wnt Signaling Pathway Gene Array (SuperArray Bioscience, Frederick, MD) which is designed to systematically profile the expression of genes involved in and downstream of Wnt signaling. The array includes the Wnt ligands and their receptors, intracellular signaling molecules, and representative target genes. Pre-resveratrol/GP samples were compared side-by-side to post-resveratrol/GP samples from the same patient to define changes in gene expression. The GEArray expression analysis software was utilized to enable background normalization, correction for different degrees of exposure, and normalization with multiple housekeeping gene controls on each membrane. Insufficient RNA was available to conduct microarray analysis from one patient $(80 \mathrm{mg}$ /day resveratrol tablets) though samples from this patient were analyzed by quantitative real-time polymerase chain reaction (PCR).

\section{Quantitative real-time PCR}

Quantitative real-time PCR (qRT-PCR) was utilized to define the expression of cylinD1, axinII, and myc. Specific primer sets for each gene were employed and expression was compared to actin controls in all cases. Primer pairs were obtained from Qiagen (Valencia, CA), catalog numbers QT00495285, QT00037639, QT00035406, and QT00095431. qRT-PCR was also utilized to examine changes in the expression of two colonic stem cell markers, CD133 and LGR5 (GPR49). We utilized a previously described primer pair for CD133: forward: 5'-GCATTGGCATCTTCTATGGTT-3', reverse: 5'-CGCCTTGTCCTTGGTAGTGT-3', ${ }^{26}$ and Quiagen primers for LGR5, catalog number QT00027720. For qRT-PCR experiments, the relative RNA expression was calculated by the comparative threshold cycle method utilizing SYBR Green reagents (Applied Biosystems) and an ABI Prism7000 Sequence Detection System. Reaction conditions for CD133: 50 cycles at $95{ }^{\circ} \mathrm{C}-30 \mathrm{~s} ; 55^{\circ} \mathrm{C}-30 \mathrm{~s} ; 60^{\circ} \mathrm{C}-30 \mathrm{~s}$. Reaction conditions for Qiagen primers: 50 cycles of $95{ }^{\circ} \mathrm{C}-15 \mathrm{~s}$; $55^{\circ} \mathrm{C}-30 \mathrm{~s} ; 60{ }^{\circ} \mathrm{C}-30 \mathrm{~s}$.

\section{In vitro analyses}

Resveratrol-induced changes in the expression of $c-m y c$ were defined by qRT-PCR in RKO colon cancer cells (American Type Culture Collection, Manassas, VA). This cell line, originally obtained from a patient with hereditary nonpolyposis colon cancer, displays microsatellite instability (MSI, or RER(+)) and has no known Wnt pathway activation. It was maintained in culture in RPMI1640 media at 5\% $\mathrm{CO}_{2}$ with $10 \%$ fetal bovine serum (FBS). Pure trans-resveratrol was obtained from Sigma-Aldrich (St. Louis, MO) and a stock solution was prepared in dimethyl sulfoxide (DMSO). Final concentrations of resveratrol for the experiments were $10 \mu \mathrm{M}$ and $20 \mu \mathrm{M}$ with $<1 \% \mathrm{v} / \mathrm{v}$ DMSO. Exposure time to resveratrol was 48 hours. The same primers that were used for the tissue-based experiments, as described above, were used.

To define the effect of GP on Wnt signal throughput in vitro, a normal colon-derived cell line, NCM460 was used. NCM460 was obtained from Incell Corporation (San Antonio, TX). ${ }^{27}$ This cell line was maintained in M3 Base cell culture medium complete (catalog number M300A-500; Incell) with 10\% FBS. For these studies, 1.3 grams of GP was extracted into $30 \mathrm{~mL}$ of $100 \%$ ethanol by a modification of the method of Cho and colleagues. ${ }^{28}$ Briefly, the GP suspension in ethanol was sonicated for five minutes using a bath type $47 \mathrm{KHz}$ Bransonic 1510 ultrasonic processor. Insoluble material was removed by centrifugation at $10,000 \times \mathrm{g}$ for 15 minutes. Ethanol extracts were stored at $-20^{\circ} \mathrm{C}$. $1-\mathrm{mL}$ aliquots were processed to dryness in a SpeedVac DNA110. Each aliquot contained approximately $16 \mathrm{mg}$ of solid material. Stock solutions of $40 \mathrm{mg} / \mathrm{ml}$ were prepared in 93\% DMSO, 7\% water.

Wnt throughput was defined using a Wnt pathway reporter construct containing seven LEF/TCF consensus binding sites preceding a secreted alkaline phosphatase (7X-SEAP) reporter. ${ }^{25}$ Activation of Wnt signaling leads to production of SEAP which is secreted into the culture media. Cells were typically between $70 \%$ to $90 \%$ confluent on the day of transfection. Reporter plasmid DNA, $1.2 \mu \mathrm{g}$ and $0.12 \mu \mathrm{g}$ of LEF1 expression construct, and/or inactive DNA (pBluescript) for a total mass of $1.44 \mu \mathrm{g}$ DNA per well was transfected using Lipofectamine 2000 (Invitrogen, Calsbad, CA) following manufacturer's recommendations. LEF1 was used to augment basal Wnt pathway activity in order to facilitate the detection of potential GP extractinduced pathway inhibition. Each condition was performed in triplicate. After 48 hours, media was recovered and $20 \mu \mathrm{L}$ 
was assayed using Great EscAPe SEAP detection kit (BD Biosciences, San Jose, CA) in a 96-well format. Plates were read on a ML3000 microtiter plate luminometer (Dynatech Laboratories, Chantilly, VA). Experiments were performed with and without $60 \mu \mathrm{g} / \mathrm{ml}$ GP extract and all experiments were performed in conjunction with transfection of a SEAP plasmid lacking LEF/TCF binding sites as a control.

\section{Statistics}

Pre- and post-resveratrol/GP expressions of individual genes by microarray were compared for all participants at each dose level on the clinical protocol with a two-tailed paired $t$-test. Composite target gene expression comparisons used a two-tailed unpaired $t$-test. Gene expression by qRT-PCR and Wnt throughput SEAP activity values were compared in individual cohorts with a two-tailed unpaired $t$-test. Levels of significance were defined as $\alpha<0.05$.

\section{Results}

\section{Clinical trial monitoring}

No patients experienced any toxicities while enrolled on the clinical trial, regardless of whether they received resveratrol tablets or the freeze-dried GP. Specifically, there were no reported changes in bowel habits (diarrhea or constipation), no abdominal bloating, no nausea or vomiting, and no gastrointestinal bleeding.

\section{Expression of Fz receptors, Wnt ligands, and Wnt inhibitors in normal mucosa and colon cancer}

The expression of Wnt ligands and numerous Wnt inhibitory genes of the soluble frizzled-related protein (sFRP), dikkopf (DKK) and Wnt inhibitory factor (WIF) families was not statistically different between normal mucosa and colon cancer (Figure 1). In cancer, several Fz receptors had increased expression, including Fz1, Fz2, Fz3, Fz4, Fz6, Fz7, and Fz10, but only the expression of Fz2 was statistically significant ( $\mathrm{p}<0.05$; Figure $1 \mathrm{~A})$. There were trends for increased Wnt ligand expression (Figure 1B) in cancer, including Wnt1, Wnt2, Wnt3a, Wnt10a, and Wnt11 and for several of the Wnt inhibitory genes (Figure 1C), but none of these reached statistical significance.

\section{Wnt target genes in normal mucosa and colon cancer}

Wnt pathway target gene expression was uniformly higher in cancer than in normal mucosa (Figure 2A). The composite target gene expression, including $L E F 1$, myc, jun, nemo, Tcf7, cyclinD1, FGF4, and axinII, was 350\% higher in cancer than in normal tissue ( $\mathrm{p}=0.002$; Figure $2 \mathrm{~B})$, indicating activated Wnt signaling in the cancer tissue. Our data also confirms minimal expression of LEF1 in normal mucosa with increased expression in colon cancer ( $p=0.07$; Figure $2 \mathrm{~A}$ ), as we have reported previously using different experimental methodologies. ${ }^{29}$ Wnt pathway activation in colon cancer compared to normal colonic mucosa was confirmed by qRT-PCR (Figure 2C) which demonstrated increased expression of cyclinDl $(\mathrm{p}=0.001)$ and $\operatorname{axin} I I(\mathrm{p}<0.001)$. The expression of myc was increased in cancer but the difference of expression compared to normal mucosa was not statistically significant.

\section{Effect of resveratrol/GP on Wnt target gene expression in colon cancer}

In cancer tissue, resveratrol/GP treatment led to no change or a slight increase in most of the Wnt pathway target genes (Figure 3). The microarray data (Figure 3A) suggested increases in $m y c$ and cyclinDl expression, with slight decreases seen for LEF1, nemo, TCF7 and FGF4. AxinII, and jun expression were unchanged. Composite target gene expression by microarray following exposure to resveratrol/GP was unchanged in colon cancer tissue when compared to the pretreatment baseline (Figure 3B). Confirmatory qRT-PCR (Figure 3C) of the cancer tissue pre- and post-resveratrol $80 \mathrm{mg} /$ day (R80) and GP $80 \mathrm{~g} /$ day (GP80) demonstrated significant increases in expression for both myc and cyclinD1. These increases were greatest following GP80 and reached statistical significance ( $\mathrm{p}<0.01$ and $\mathrm{p}<0.05$, respectively).

\section{Effect of resveratrol/GP on Wnt target gene expression in normal mucosa}

In normal colonic mucosa, resveratrol/GP treatment resulted in a decrease in most of the Wnt target genes evaluated by microarray (Figure 4A), including myc, jun, TCF7, axinII, and cyclinD1. The composite target gene expression by microarray in the normal mucosa was significantly reduced following exposure to resveratrol/GP (Figure 4B) when all treatment groups were combined $(p=0.03)$ though the most significant reduction in Wnt target gene expression was seen at the lower dose of GP (GP80, p < 0.001). The reduction in cyclinDl $(\mathrm{p}<0.005)$ and $\operatorname{axinII}(\mathrm{p}<0.05)$ following exposure to GP80 were confirmed by qRT-PCR (Figure 4C). No significant differences were seen in cyclinDI and axinII expression following exposure to R80. The one 
A

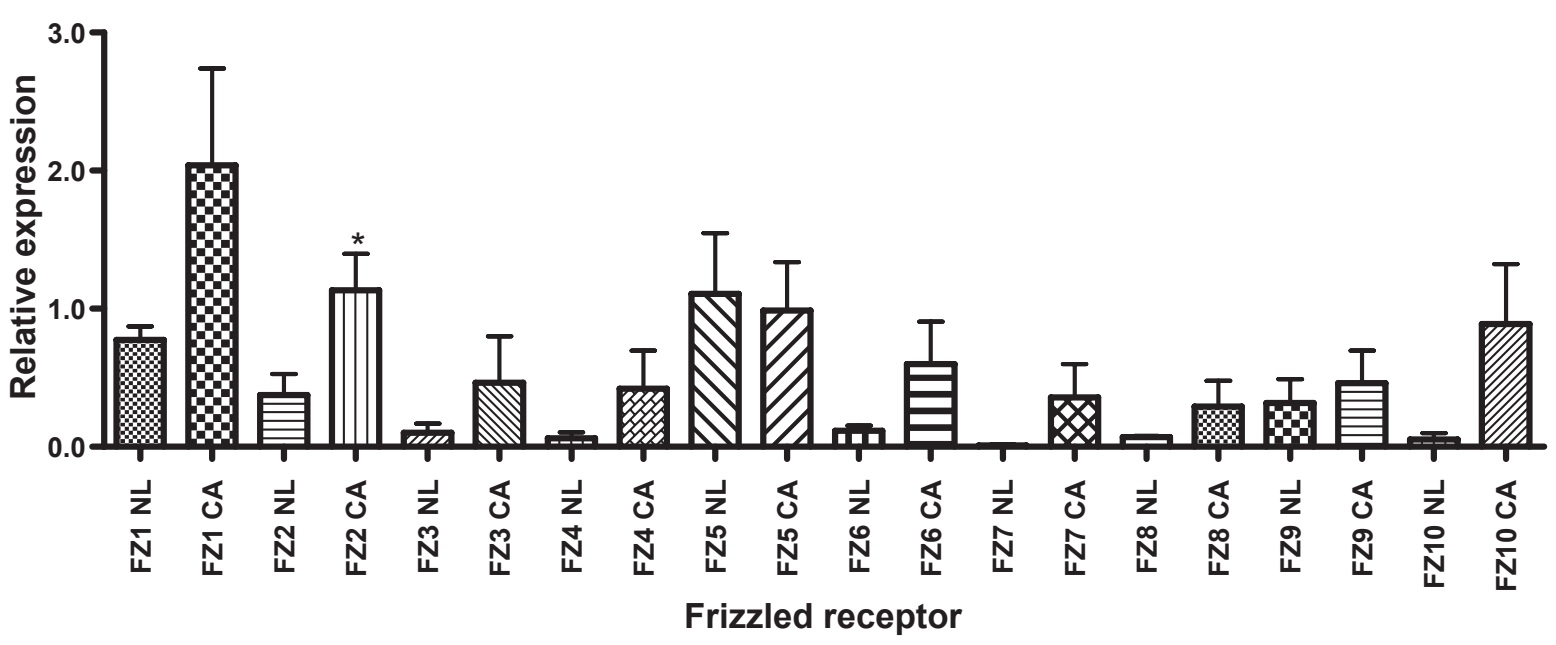

B

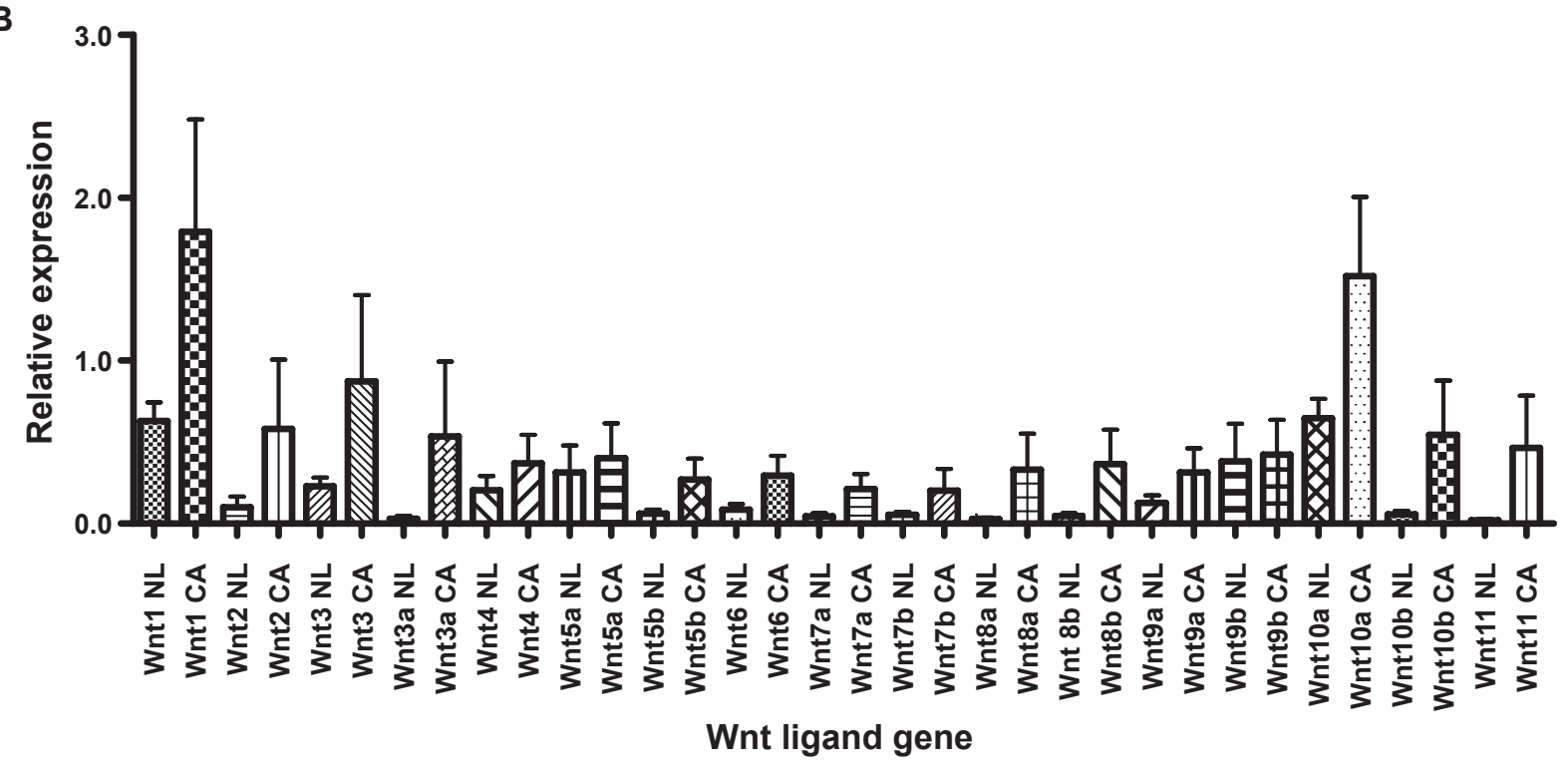

C

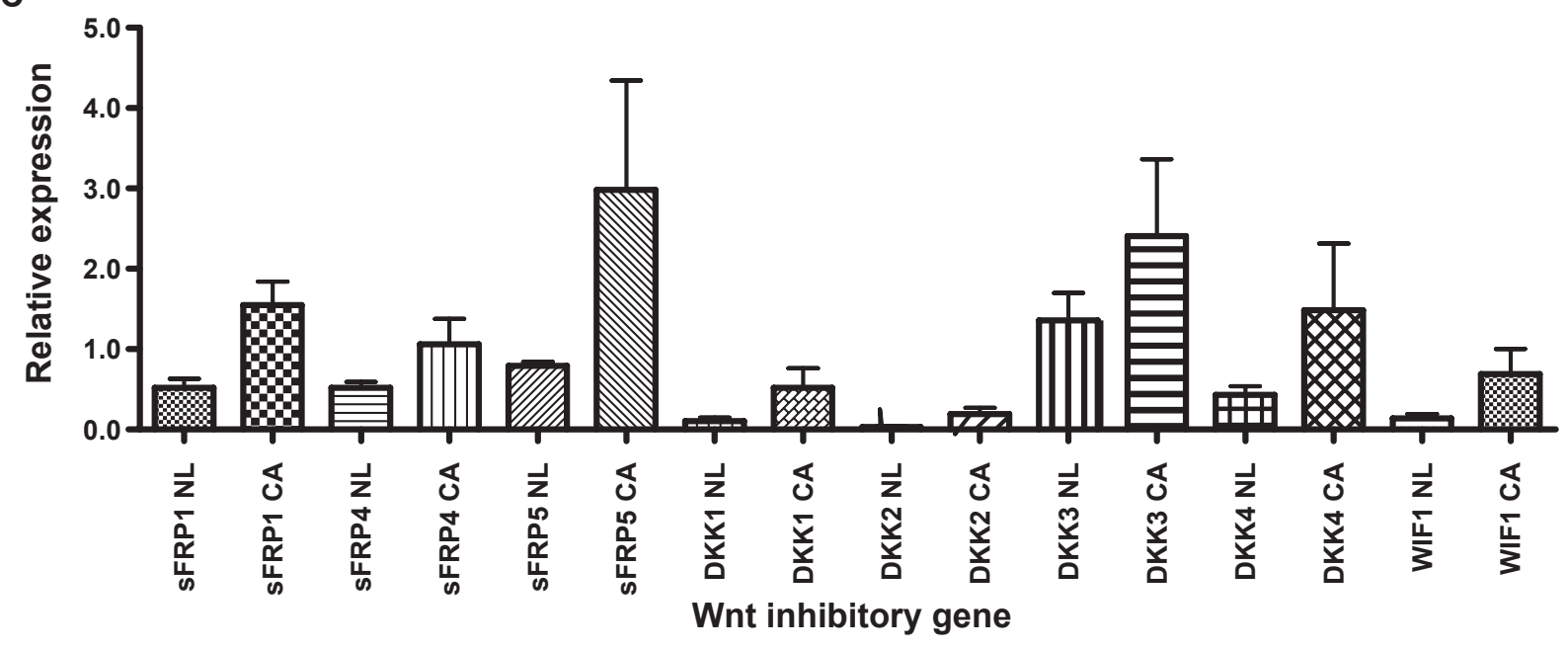

Figure I Microarray gene expression results for all patients contrasting expression levels in normal mucosa (NL) and colon cancer (CA) tissues. Frizzled (FZ) receptor expression is depicted in panel $\mathbf{A}, \mathrm{Wnt}$ ligand expression is depicted in panel $\mathbf{B}$, and the expression of various Wnt inhibitory molecules are depicted in panel $\mathbf{C}$. Abbreviations: sFRP, soluble frizzled-related protein; DKK, dikkopf; WIFI, Wnt inhibitory factor I.

Notes: $*_{p}<0.05$, without correction for multiplicity. 

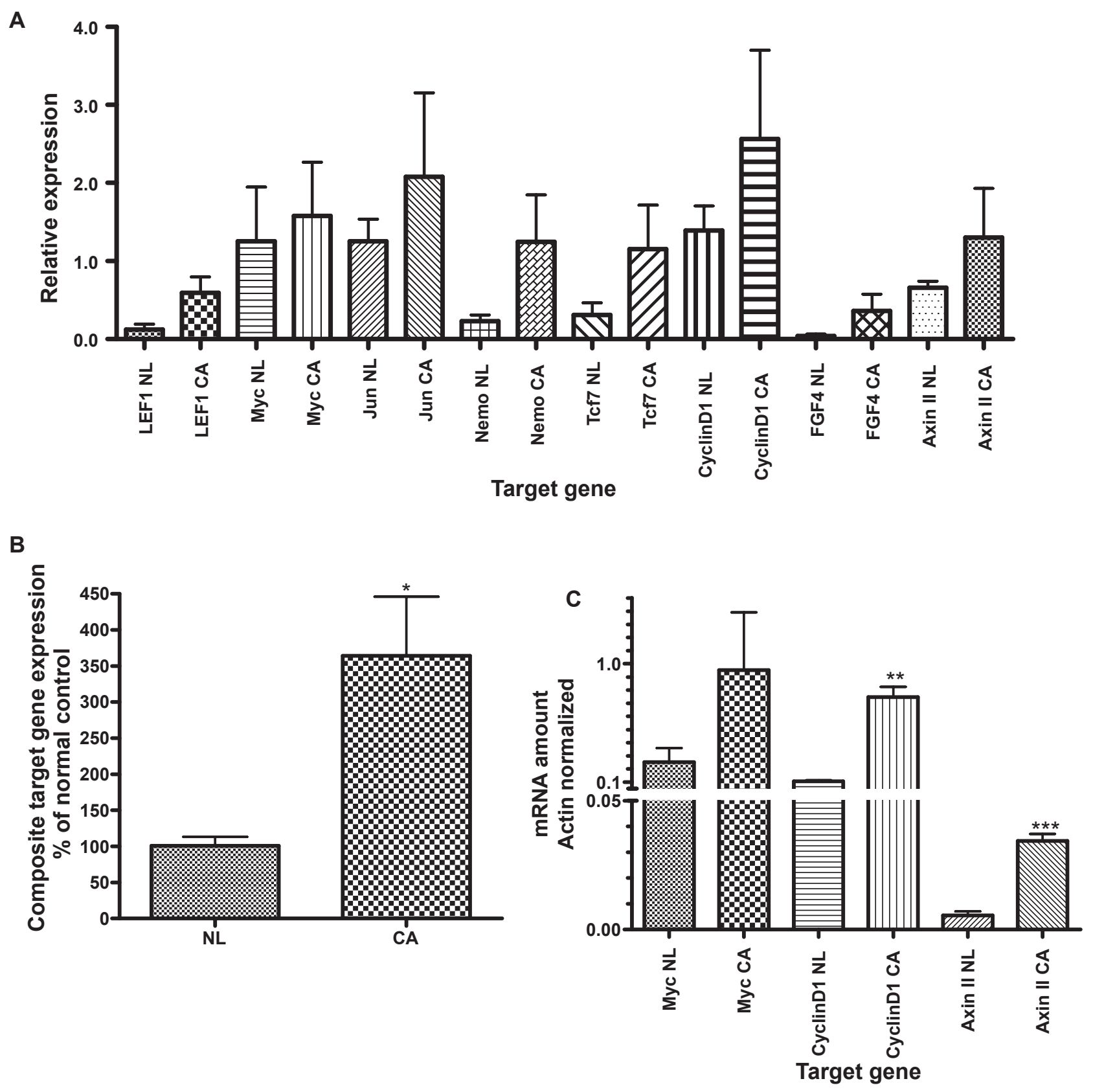

Figure 2 Panel A: Expression of Wnt target genes by microarray, contrasting normal mucosa (NL) and colon cancer (CA). Panel B: Composite target gene expression (microarray data), normalized to percent of normal mucosa. Panel C: Target gene expression by quantitative real-time polymerase chain reaction for myc, cyclinDI-, and axinll, contrasting normal mucosa and cancer.

Notes: ${ }^{*} p<0.002$. ${ }^{*} p=0.001$. ${ }^{* * *} p<0.001$.

target gene for which a decrease in expression following GP80 could not be confirmed was $m y c$. By qRT-PCR, $m y c$ was paradoxically increased $(\mathrm{p}=0.01)$.

\section{Effect of resveratrol/GP on stem cell markers}

Because the greatest reduction in Wnt signaling appeared to occur following exposure to GP80, and because colonic stem cell proliferation is regulated in part by Wnt signals, the effect of GP80 on two colonic stem cell markers, CD133 and LGR5 (GPR49), was evaluated by qRT-PCR (Figure 5). Overall, CD133 and LGR5 expression was higher in cancer than in normal mucosa $(\mathrm{p}<0.05$ and $\mathrm{p}<0.005$, respectively, data not shown) and no significant change in expression was seen following GP80. However, following exposure to GP80, CD133 expression in normal mucosa 


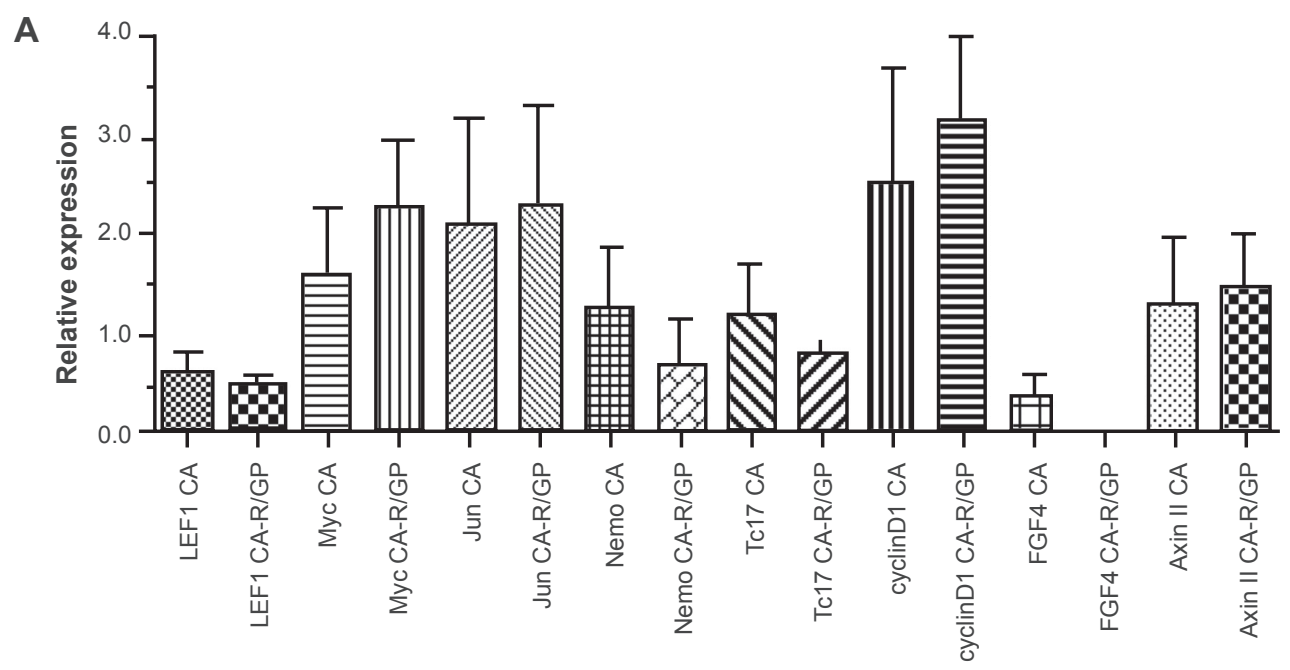

Target gene; Colon cancer

\section{B}

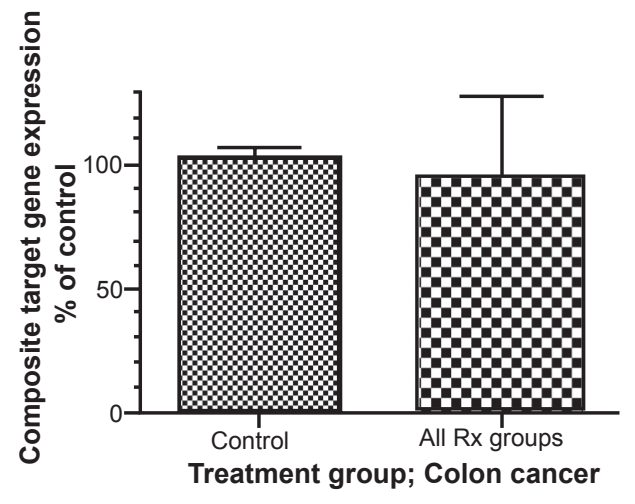

C

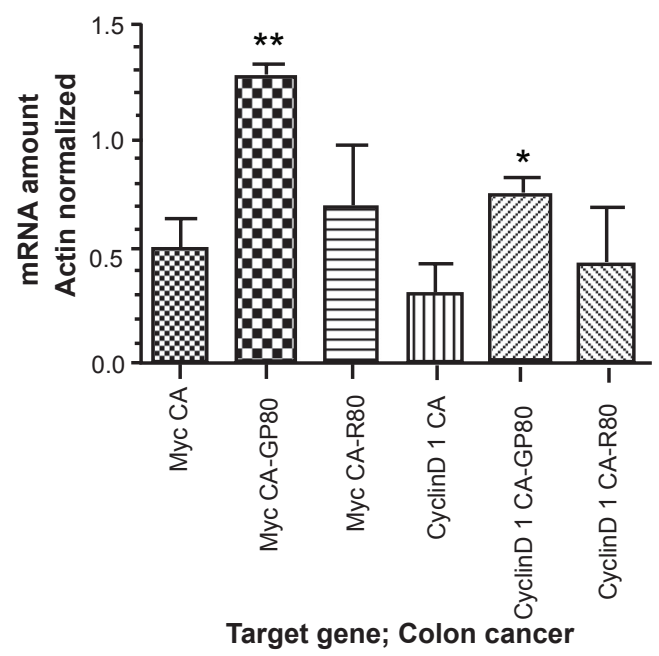

Figure 3 Target gene expression in colon cancer prior to (CA) and following (CA-R/GP) exposure to resveratrol or freeze-dried grape powder. Panel A depicts microarray data from all patients. Panel B depicts composite target gene expression (microarray data), normalized to percent of control, overall. No statistically significant difference is present between the two groups. Panel $\mathbf{C}$ depicts myc and cyclinD I expression by quantitative real-time polymerase chain reaction following exposure to resveratrol at $80 \mathrm{mg} /$ day or freeze-dried grape powder at $80 \mathrm{~g} /$ day. R80, resveratrol $80 \mathrm{mg} /$ day; GP80, grape powder $80 \mathrm{~g} /$ day. Notes: ${ }^{*} \mathrm{p}<0.05$. **p $<0.01$.

was decreased $(\mathrm{p}<0.05)$ as was the expression of LGR5 (trend, $\mathrm{p}=0.09$ ).

\section{In vitro effect of resveratrol on myc expression}

Because the effect of resveratrol/GP on myc expression was paradoxically opposite of its effect on other Wnt target genes, we examined the effect of resveratrol on myc expression in RKO colon cancer cells in vitro. Resveratrol is known to inhibit Wnt signaling in RKO cells in vitro at concentrations as low as $10 \mu \mathrm{M}$ and $20 \mu \mathrm{M} .{ }^{25}$ By qRT-PCR, myc expression increased in a dose-dependent fashion following exposure to $10 \mu \mathrm{M}$ and $20 \mu \mathrm{M}$ resveratrol to over $300 \%$ of control (Figure 6A).

\section{In vitro effect of GP on Wnt signal throughput}

Because the reduction in Wnt target gene expression was greatest for GP80, which contained the lowest amounts of resveratrol in any of the treatment groups but contained numerous bioactive components not present within the plantderived resveratrol formulation, the ability of GP to directly 


\section{A}

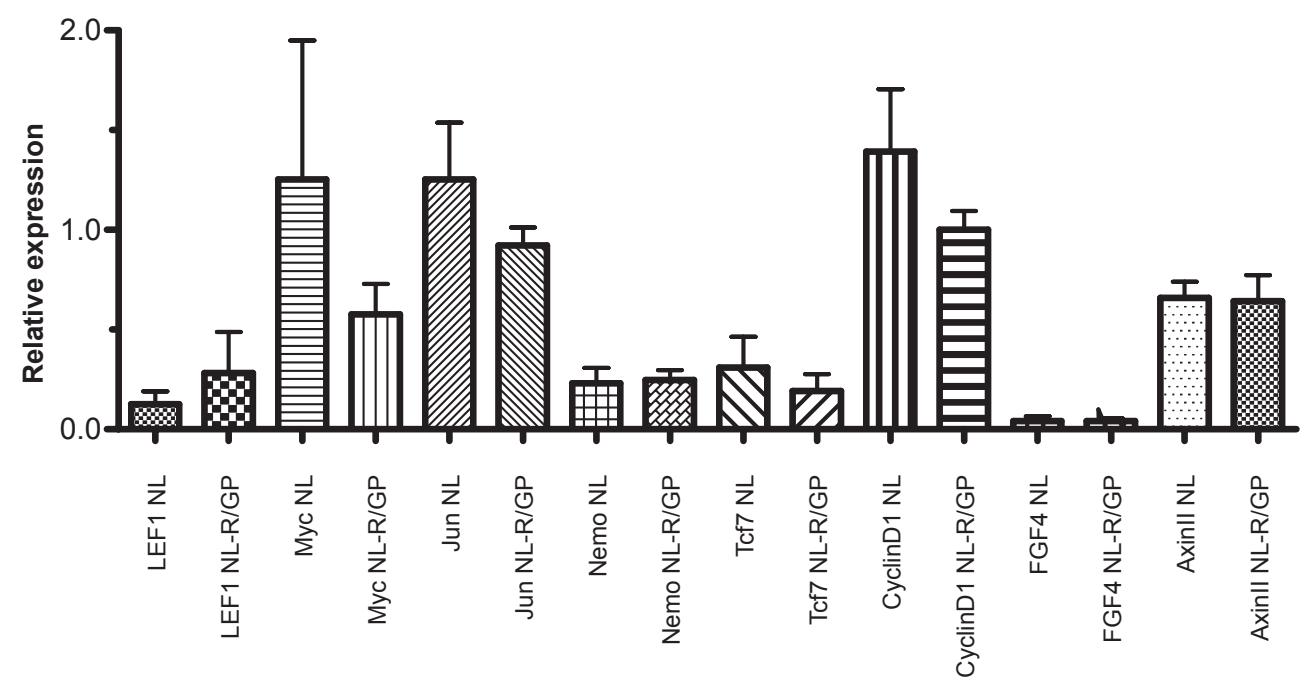

Target gene; Normal mucosa

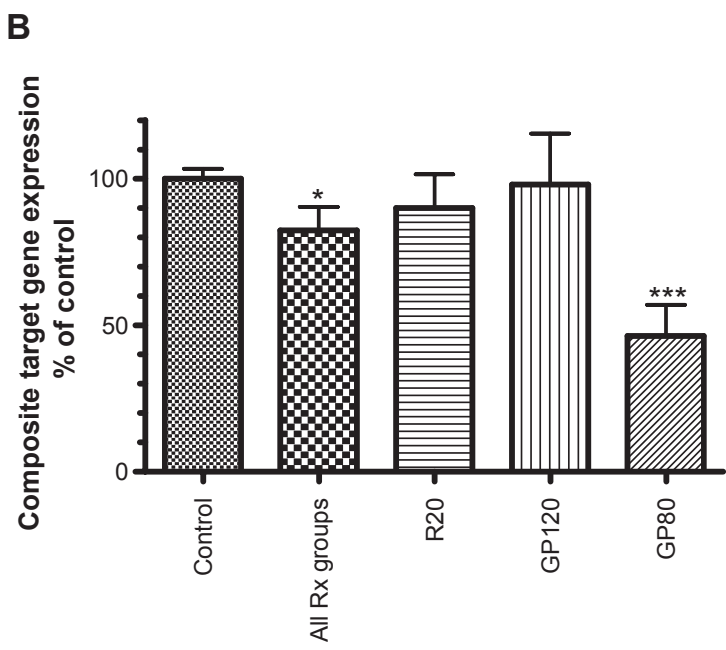

Treatment group; Normal mucosa

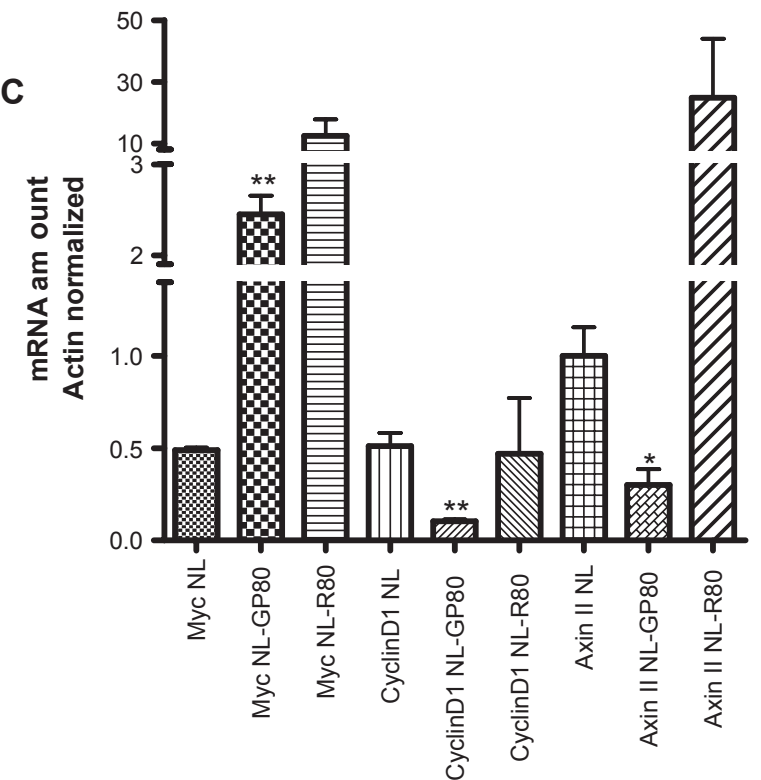

Target gene; Normal mucosa

Figure 4 Target gene expression in normal colonic mucosa prior to (NL) and following (NL-R/GP) exposure to resveratrol or freeze-dried grape powder. Panel A depicts microarray data from all patients. Panel B depicts composite target gene expression (microarray data), normalized to percent of control, overall and for each individual dose cohort. Panel C depicts myc, cyclinD I and axinll expression by quantitative real-time polymerase chain reaction. R20, resveratrol $20 \mathrm{mg} /$ day; R80, resveratrol $80 \mathrm{mg} /$ day; GPI20, grape powder at $120 \mathrm{~g} /$ day; GP80, grape powder at $80 \mathrm{~g} /$ day. Notes: ${ }^{*} \mathrm{p}<0.05$. ${ }^{* *} \mathrm{p}<0.01$. ${ }^{* * *} \mathrm{p}<0.001$.

inhibit Wnt signaling in vitro was evaluated with a reporter construct containing LEF/TCF binding sites. Additionally, since the effect of GP80 in the clinical trial was confined to normal colonic mucosa, the normal colon-derived cell line NCM460 was used for these experiments. As can be seen in Figure 6B, GP significantly inhibited Wnt pathway throughput to nearly half of the baseline activity in NCM460 cells $(\mathrm{p}<0.005)$.

\section{Discussion and conclusions}

Based on the microarray results from patients enrolled onto the clinical trial, no major differences in the expression between normal colonic mucosa and colon cancer were seen for the frizzled receptors, Wnt ligands or soluble Wnt inhibitory molecules, with the possible exception of Fz2 which exhibited higher levels of expression in cancer tissue. Several of these molecules exhibited trends toward differential 


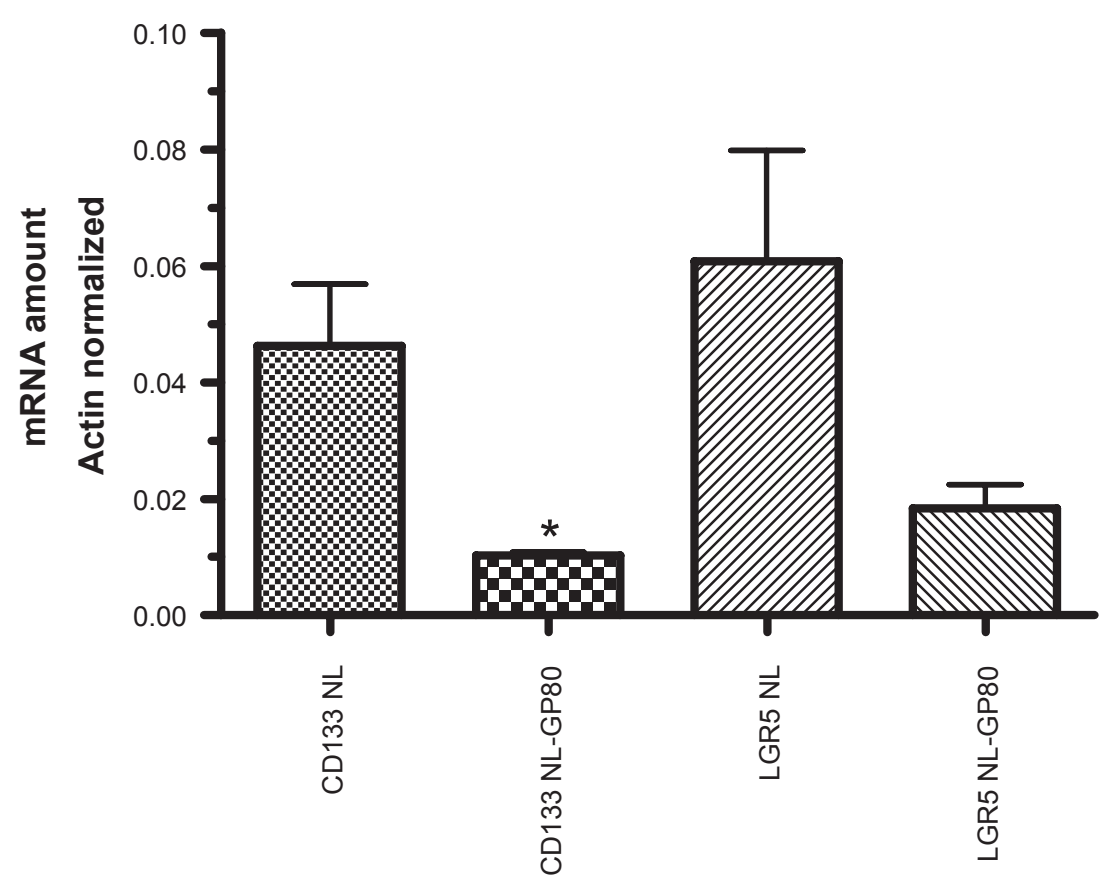

\section{Stem cell marker}

Figure 5 Expression of colonic stem cell markers CDI33 and LGR5 (GPR49) in normal colonic mucosa prior to (NL) and following (NL-GP80) exposure to freeze-dried grape powder at $80 \mathrm{~g} /$ day as measured by quantitative real-time polymerase chain reaction.

Notes: *p $<0.05$ for CDI33 NL vs CDI 33 NL-GP80. $p=0.09$ for LGR5 NL vs LGR5 NL-GP80.

expression which could be investigated by more sensitive methodologies such as qRT-PCR in future studies. Of note, LEF1, a downstream transcriptional regulator for the Wnt pathway, was minimally expressed in normal mucosa but showed increased expression in cancer which is consistent with prior observations. ${ }^{29,30}$ In addition to being an integral component of the pathway, LEF1 is also a Wnt target gene ${ }^{31}$ which may account for the increased expression in cancer that was observed here.

Our microarray data of Wnt target gene expression suggests that colon cancers exhibit increased Wnt throughput compared to normal mucosa, consistent with the known activation of Wnt signaling in the majority of colorectal cancers. ${ }^{21}$ These findings were confirmed with qRT-PCR of cyclinD1 and axinII, two well characterized Wnt target genes. ${ }^{32,33}$ Colon cancers also exhibited higher levels of expression of CD133, which is purported to be a marker of colon cancer stem cells ${ }^{34}$ and is, itself, a Wnt target gene. Interestingly, no differences in myc expression between normal and cancer could be confirmed by qRT-PCR.

Patients treated with resveratrol/GP had no change overall in the composite Wnt target gene expression in colon cancer. Several of the genes, including myc and cyclinD1, increased in the cancer tissue following the two-week exposure to GP80, though no statistically significant change was seen following exposure to R80. The mechanism for these increases is unclear and will necessitate further investigation and also caution in promoting the use of plant-derived resveratrol or resveratrol foodstuffs with therapeutic intent for colon cancer patients. Additionally, target gene expression is only an indirect indicator of Wnt signal throughput and even if Wnt signaling was suppressed, other regulatory pathways may be responsible for increases in $m y c$ and cyclinD1 expression. Alternatively, cancer therapeutic-oriented effects might also be seen at higher concentrations obtained with pharmacologic dosages of pure trans-resveratrol. Certainly in vitro, ${ }^{5}$ high concentrations of resveratrol result primarily in proliferationinhibiting and apoptosis-inducing effects.

Resveratrol and GP appear to have quite a different effect on normal colonic mucosa, where inhibition of Wnt target gene expression is seen by microarray and confirmed by qRT-PCR of cyclinDl and axinII. The greatest effect was seen with GP80 which had the lowest resveratrol content of all the cohorts but many other bioactive components not present in the plant-derived resveratrol capsules. This amount of GP is equivalent to ingestion of $0.45 \mathrm{~kg}$ of fresh grapes per day, or approximately three USDA 
A

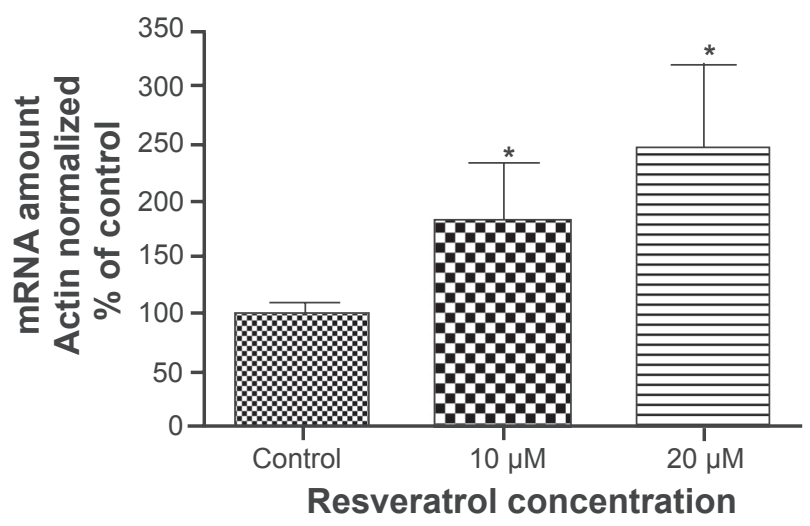

B

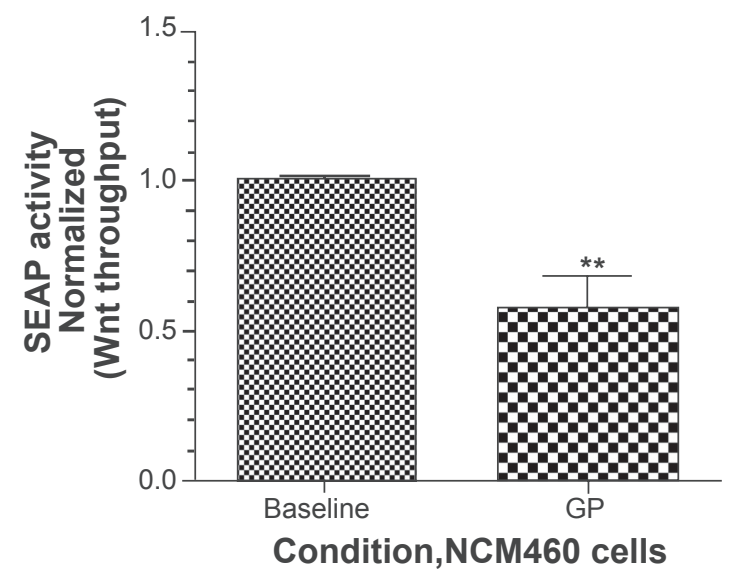

Figure 6 PanelA: myc expression by quantitative real-time polymerase chain reaction in RKO colon cancer cells at baseline and following exposure to $10 \mu \mathrm{M}$ and $20 \mu \mathrm{M}$ resveratrol in vitro. Panel B: Wnt throughput as measured by secreted alkaline phosphatase (SEAP) activity following transfection of the 7X-SEAP LEF/TCF reporter construct (see methods) in normal mucosa-derived NCM460 cells, at baseline and following exposure to reconstituted grape powder (GP).

Notes: ${ }^{*} \mathrm{p}<0.05$. ${ }^{*} \mathrm{p}<0.005$.

servings. This is an amount which is dietarily achievable and represents an extremely low dose of resveratrol itself. Expression of both CD133 and LGR5, markers of colon cancer and colonic stem cells, and Wnt target genes, ${ }^{34,35}$ decreased in normal mucosa following exposure to GP80 though the change in LGR5 did not reach statistical significance. The influence of GP on Wnt signal throughput was tested directly in vitro with normal mucosa-derived NCM460 cells. After 48 hours of incubation, significant inhibition of Wnt signal throughput was noted. This is despite an extremely low resveratrol concentration in the reconstituted GP ( $4 \mu \mathrm{M} / \mathrm{kg}$ dry weight). The greater effect seen with GP, compared to resveratrol, suggests that the effect on Wnt target gene expression and Wnt signal throughput is due to coopertivity of resveratrol with other bioactive flavonoids and polyphenols in grapes, rather than to resveratrol alone. In addition, the Wnt inhibitory effect in normal mucosa suggests that GP or resveratrolcontaining foods may have more clinical utility for colon cancer prevention rather than for treatment of established colon cancer.

Based on our results, myc does not appear to be a good marker for Wnt pathway activation, possibly because of potential effects of resveratrol on other pathways involved in regulation of myc expression such as those mediated by $\mathrm{NF}-\mathrm{KB}$ and or initiated following activation of tyrosine kinase growth factor receptors. ${ }^{36}$ An increase in $m y c$ was confirmed in vitro in colon cancer cells with low concentrations of trans-resveratrol which are known to inhibit Wnt signal throughput. ${ }^{25}$ Interestingly, while increased myc expression would generally be considered detrimental in cancer, due to its pro-proliferative effects, increased expression in normal mucosa, where cells have intact p53-mediated apoptotic pathways, can result in induction of apoptosis rather than proliferation. ${ }^{37}$ Further study of the colonic mucosal proliferative response to resveratrol or GP, with correlation to myc and cyclinDl expression levels, are indicated.

There are several characteristics of this study which dictate caution before generalizing the findings. This was a pilot study with a small number of participants which could account for variability in the expression levels of molecular markers as well as the lack of statistically significant alterations observed. For the microarray data, multiple comparisons were performed for individual groups so the level of statistical stringency is critical, though confirmation by qRTPCR partially mediates this concern. No attempt was made to control for dietary intake of the participants during the two-week treatment period and no food recall to determine whether participants ingested resveratrol-rich foods during this period was performed. Additionally, it was not recorded whether other confounding medications which might affect the Wnt pathway, such as nonsteroidal anti-inflammatory medications, were ingested.

Despite these limitations, this is the first reported trial of resveratrol/GP in cancer patients. Low dosages of resveratrol in combination with other bioactive compounds in GP inhibit Wnt signaling in normal colonic mucosa as indicated by a reduction in the expression of a panel of Wnt target genes. This suggests that resveratrol or GP may play a beneficial role in colon cancer prevention. Further study of dietary supplementation with resveratrol and/or whole grapes or freeze-dried GP as a potential colon cancer preventive strategy is warranted. 


\section{Author contributions}

Anthony V Nguyen coordinated patient enrollment, specimen acquisition, and performed microarray assays. Micaela Martinez performed quantitative real-time PCR analyses. Michael J Stamos performed patient identification and specimen acquisition. Mary P Moyer provided NCM460 cell line. Kestutis Planutis and Christopher Hope assisted with all laboratory assays. Randall F Holcombe was responsible for the overall study design and supervision, including clinical and basic laboratory components.

\section{Acknowledgments}

This work was supported by grants to RFH from the California Table Grape Commission, the UCLA Clinical Nutrition Research Unit (CA-42710) and grant CA-82450. The authors thank Sonya Sakowsky and Allison Crase for their assistance with the clinical trial.

\section{Disclosure}

The authors declare that they have no competing interests.

\section{References}

1. American Cancer Society, Cancer facts and figures, 2007. Accessed on Mar 1, 2009. Available from: http://www.cancer.org

2. Mason JB. Nutritional chemoprevention of colon cancer. Sem on Gastrointest Dis. 2002;13:143-153.

3. World Cancer Research Fund and American Institute for Cancer Research. Food, Nutrition, Physical Activity, and the Prevention of Cancer: A Global Perspective. Washington: DC; 2007. p. 280-289.

4. Kushi LH, Byers T, Doyle C, et al; the American Cancer Society 2006 Nutrition and Physical Activity Guidelines Advisory Committee. American Cancer Society guidelines on nutrition and physical activity for cancer prevention: Reducing the risk of cancer with healthy food choices and physical activity. CA Cancer J Clin. 2006;56:254-281.

5. Szende B, Tyihak E, Kiraly-Veghely Z. Dose-dependent effects of resveratrol on proliferation and apoptosis in endothelial and tumor cell cultures. Exp Mol Med. 2000;32:88-92.

6. Tessitore L, Davit A, Sarotto I, Caderni G. Resveratrol depresses the growth of colorectal aberrant crypt foci by affecting bax and p $21^{\mathrm{CIP}}$ expression. Carcinogenesis. 2000;21:1619-1622.

7. Sengottuvelan M, Viswanathan P, Nalini N. Chemopreventive effect of trans-resveratrol - a phytoalexin against colonic aberrant crypt foci and cell proliferation in 1,2-dimethylhydrazine induced colon carcinogenesis. Carcinogenesis. 2006;27:1038-1046.

8. Sengottuvelan M, Nalini N. Dietary supplementation of resveratrol suppresses colonic tumor incidence in 1,2 dimethylhydrazine-treated rats by modulating biotransforming enzyemes and aberrant crypt foci development. Br J Nutr. 2006;96:145-153.

9. Schneider Y, Duranton B, Gosse F, Schleiffer R, Seiler N, Raul F. Resveratrol inhibits intestinal tumorigenesis and modulates hostdefense-related gene expression in an animal model of human familial adenomatous polyposis. Nutr Cancer. 2001;39:102-107.

10. Ziegler C, Rainwater L, Whelan J, McEntee MF. Dietary resveratrol does not affect intestinal tumorigenesis in $\mathrm{Apc}^{\mathrm{Min} /+}$ mice. J Nutr. 2004;134:5-10

11. Gignac EA, Bourquin LD. Influence of resveratrol and sulindac on intestinal tumor numbers in Min mice. FASEB J. 2001;15:A630.
12. Sale S, Tunstall RG, Ruparelia KC, Potter GA, Steward WP, Gescher AJ. Comparison of the effects of the chemopreventive agent resveratrol and its synthetic analog trans 3,4,5,4-tetramethoxystilbene (DMU-212) on adenoma development in the $\mathrm{Apc}^{\mathrm{Min} /+}$ mouse and cyclooxygenase-2 in human derived colon cancer cells. Int J Cancer. 2005;115: 194-201.

13. Fontecave M, Lepoivre M, Elleingand E, Gerez C, Guittet O. Resveratrol, a remarkable inhibitor of ribonucleotide reductase. FEBS Lett. 1998;421:277-279.

14. Stivala LA, Savio M, Carafoli F, et al. Specific structural determinants are responsible for the antioxidant activity and the cell cycle effects of resveratrol. J Biol Chem. 2001;276:22586-22594.

15. Khanduja KL, Bhardwaj A, Kaushik G. Resveratrol inhibits N-nitrosodiethylamine-induced ornathine decarboxylase and cyclooxygenase in mice. J Nutr Sci Vitaminol (Tokyo). 2004;50:61-65.

16. Holmes-McNary M, Baldwin AS Jr. Chemopreventive properties of trans-resveratrol are associated with inhibition of activation of the I-k-B kinase. Cancer Res. 2000;60:3477-3483.

17. Kundu JK, Shin YK, Kim SH, Surh YJ. Resveratrol inhibits phorbol ester-induced expression of COX-2 and activation of NF-kappaB in mouse skin by blocking IkappaB kinase activity. Carcinogenesis. 2006;27:1465-1474.

18. Bauer JA, Sinclair DA. Therapeutic potential of resveratrol: the in vivo evidence. Nat Rev Drug Discov. 2006;5:493-506.

19. Gescher AJ, Steward WP. Relationship between mechanisms, bioavailability and preclinical chemopreventive efficacy of resveratrol: A conundrum. Cancer Epidemiol Biomarkers Prev. 2003;12: 953-957.

20. Boocock DJ, Faust GE, Patel KR, et al. Phase I dose escalation pharmacokinetic study in healthy volunteers of resveratrol, a potential cancer chemopreventive agent. Cancer Epidemiol Biomarkers Prev. 2007; 16:1246-1252.

21. Robbins DH, Itzkowitz SH. The molecular basis of colon cancer. Med Clin NA. 2002;86:1467-1495.

22. Polakis P. The many ways of Wnt in cancer. Curr Opin Genet Dev. 2007; 17:45-51.

23. Reya T, Clevers H. Wnt signaling in stem cells and cancer. Nature. 2005;434:843-850

24. Johal KS, Woo J, Santoso C, et al. Low dose resveratrol inhibits Wnt signaling proximal to $\beta$-catenin. Proc Am Assoc Cancer Res. 2007; 48:350.

25. Hope C, Planutis K, Planoutiene M, et al. Low concentrations of resveratrol inhibit Wnt signal throughput in colon-derived cells: Implications for colon cancer prevention. Mol Nutr Food Res. 2008;52 (Suppl 1):S52-S61.

26. Liu G, Yuan X, Zeng Z, et al. Analysis of gene expression and chemoresistance of CD133+ cancer stem cells in glioblastoma. Mol Cancer. 2006;5:67.

27. Moyer MP, Stauffer JS, Manzano LA, Tanzer LR, Merriman RL. NCM460, A normal human colon mucosal epithelial cell line in vitro cell dev biol. Animal. 1996;32:315-317.

28. Cho YJ, Hong JY, Chun HS, Lee SK, Min HY. Ultrasonicationassisted extraction of resveratrol from grapes. J Food Eng. 2006;77: 725-730.

29. Hovanes K, Li TW, Munguia JE, et al. Beta-catenin sensitive isoforms of lymphoid enhancer factor-1 are selectively expressed in colon cancer. Nat Genetics. 2001;28:53-57.

30. van der Flier LG, Sabates-Bellver J, Oving I, et al. The intestinal Wnt/ TCF signature. Gastroenterology. 2007;132:628-632.

31. Li TW, Ting JH, Yokoyama NN, Bernstein A, van de Wetering M, Waterman ML. Wnt activation and alternative promoter repression of LEF1 in colon cancer. Mol Cell Biol. 2006;26:5284-5299.

32. Testu O, McCormick F. Beta-catenin regulates the expression of cyclin D1 in colon carcinoma cells. Nature. 1999;398:422-426.

33. Jho EH, Zhang T, Domon C, Joo CK, Freund JN, Costantini F. Wnt/beta-catenin/tcf signaling induces the transcription of Axin 2, a negative regulator of the signaling pathway. Mol Cell Biol. 2002;22: $1172-1183$. 
34. Nikolova T, Wu M, Brumbarov K, et al. Wnt conditioned media differentially affect the proliferation and differentiation of cord blood-derived CD133+ cells in vitro. Differentiation. 2007;75:100-111.

35. Barker N, van Es JH, Kuipers J, et al. Identification of stem cells in small intestine and colon by marker gene Lgr5. Nature. 2007;449: 1003-1008.
36. Liu J, Levens D. Making myc. Curr Top Microbiol Immunol. 2006;302:1-32.

37. Nilsson JA, Cleveland JL. Myc pathways provoking cell suicide and cancer. Oncogene. 2003;22:9007-9021. 
